\title{
Assessment of the Subjective Benefit of Electric Acoustic Stimulation with the Abbreviated Profile of Hearing Aid Benefit
}

\author{
Wolfgang K. Gstoettner ${ }^{\mathrm{a}}$ Paul Van de Heyning ${ }^{\mathrm{d}} \quad$ Alec Fitzgerald O'Connor ${ }^{\mathrm{e}}$ Jan Kiefer ${ }^{f}$ \\ Constantino Morera ${ }^{i}$ Manuel Sainz ${ }^{j}$ Katrien Vermeire ${ }^{b}$ Sonelle McDonald ${ }^{e} \quad$ Laura Cavallé $^{i}$ \\ Juan García Valdecasas ${ }^{j}$ Oliver F. Adunkak Uwe Baumanng ${ }^{\mathrm{k}}$ Andrea Kleine-Punte ${ }^{\mathrm{d}}$ \\ Hanna Brockmeier h, I Ilona Anderson ${ }^{c}$ Silke Helbig ${ }^{g}$ \\ ${ }^{a}$ Department of Otolaryngology Head and Neck Surgery, AKH Vienna, Vienna, ${ }^{b}$ Christian Doppler Laboratory \\ for Active Implantable Systems, Institute of Ion Physics and Applied Physics, University of Innsbruck, and \\ ${ }^{c}$ Clinical Research Department, MED-EL, Innsbruck, Austria; ${ }^{d}$ Department of Otorhinolaryngology, Head and \\ Neck Surgery, Antwerp University Hospital, University of Antwerp, Antwerp, Belgium; 'Auditory Implant Clinic, \\ St. Thomas' Hospital, London, UK; ${ }^{f} \mathrm{HNO}-Z e n t r u m$ Regensburg, Regensburg, ${ }^{9}$ Department of Otorhinolaryngology, \\ Goethe University, Frankfurt, and h Department of Otolaryngology, Head and Neck Surgery, Technical University \\ Munich, Munich, Germany; 'Servicio ORL, Hospital Universitario La Fe, Valencia, and 'Servicio de Otorrino, \\ Facultad de Medicina de Granada/Hospital San Cecilio, Granada, Spain; ' Department of Otolaryngology, Head and \\ Neck Surgery, University of North Carolina at Chapel Hill, Chapel Hill, N.C., USA; 'Department of Otolaryngology, \\ Head and Neck Surgery, University of Basel, Basel, Switzerland
}

\section{Key Words}

Abbreviated Profile of Hearing Aid Benefit questionnaire • Electric-acoustic stimulation - Hearing preservation • Ski slope hearing loss $\cdot$ Cochlear implant $\cdot$ Subjective benefit

\begin{abstract}
Conclusion: This study demonstrates that electric-acoustic stimulation (EAS) significantly decreases the subjective impairment in speech perception. Objectives: To assess the subjective benefit of EAS over the first 12 months after EAS fitting using the Abbreviated Profile of Hearing Aid Benefit (APHAB). Method: Twenty-three EAS users, implanted with either the PULSAR ${ }_{\mathrm{Cl}}{ }^{100}$ FLEX $^{E A S}$ provided with the DUET EAS processor or the COMBI40+ Medium provided with the TEM$\mathrm{PO}+$ speech processor, were included. Electric stimulation was activated about 1 month postoperatively; ipsilateral acoustic stimulation was added 2 months thereafter. EAS
\end{abstract}

benefit was measured preoperatively with only a hearing aid and postoperatively at EAS fitting and then 3, 6 and 12 months after EAS fitting using the APHAB. Results: Subjects reported significant improvements in the global score with a mean decrease in impairment from $74 \%$ preoperatively to $45 \%$ after 3 months of EAS use. Furthermore, clinical relevance was demonstrated in multiple subscales between preoperative and first fitting reflecting a true benefit of EAS with a probability of $95 \%$.

Copyright $\odot 2011$ S. Karger AG, Basel

\section{Introduction}

The combination of electric and acoustic hearing in the same ear, also known as electric-acoustic stimulation $\left(\mathrm{EAS}^{\circledR}\right)$, is a relatively new treatment method for individuals with a ski slope type hearing loss mainly affecting the high frequencies who gain minimal or no benefit

\section{KARGER}

Fax +4161306 1234 E-Mail karger@karger.ch www.karger.com
(ㄷ) 2011 S. Karger AG, Basel

0301-1569/11/0736-0321\$38.00/0

Accessible online at:

www.karger.com/or
Prof. Wolfgang Gstoettner, MD

Medical University of Vienna

Department of Otolaryngology, Head and Neck Surgery

Waehringer Guertel 18-20, AT-1090 Vienna (Austria)

Tel. +43 140400 3305, E-Mail wolfgang.gstoettner@ meduniwien.ac.at 
from traditional instrument amplification. EAS was first performed in 1999 [1] and is now a routinely used treatment method. The main focus of EAS is based on the use of residual acoustic hearing in the low frequencies. It is thus of crucial importance during EAS surgery to prevent any damage to the apical low-frequency regions of the cochlea by means of, for example, a limited insertion depth, shorter electrodes or flexible electrodes specifically designed for hearing preservation [1-3].

When residual hearing is successfully preserved during cochlear implantation, audiological outcome measures demonstrate that EAS users have a marked benefit and that a strong synergistic effect of using both electric and acoustic devices exists, which is particularly noticeable in speech understanding in noise $[2,4-7]$ as well as in music perception [8].

In addition to these audiological outcome measures obtained by objective testing, subjective benefits are also of great importance. And, although the subjectively perceived benefit generally corresponds with and supports the objectively measured improved speech perception, benefits seen in speech perception testing may not account for every aspect of subjective benefits. Studies (as reported in Cox et al. [9]) have shown that subjectively rated improvement in speech understanding, attributed to the hearing aid, accounts for less than $40 \%$ of the variance in satisfaction regarding the hearing aid. This result suggests that user satisfaction is in fact also greatly impacted by other issues such as acoustic feedback, dexterity or user expectations.

To evaluate subjective benefits, patient self-report surveys [10] or questionnaires such as the Glasgow hearing aid benefit profile [11] or the Abbreviated Profile of Hearing Aid Benefit (APHAB) $[12,13]$ are generally used. The APHAB questionnaire, as used in the present study, was designed for hearing-impaired patients to report the amount of trouble they are having with communication and noise in everyday situations. In the past, multiple studies have already been using the APHAB to obtain such subjective results $[14,15]$. However, since EAS is still a relatively new treatment method, only few studies have been done so far using the APHAB to investigate the subjective benefits of EAS. Helbig et al. [16] performed such a study, using the APHAB questionnaire, amongst speech perception tests, to evaluate the subjective benefit of a cochlear implant (CI)/EAS user upgrade to a combination of hearing aid and speech processor in one single device (DUET ${ }^{\mathrm{TM}}$ ). Before the upgrade, 6 of the 9 study subjects were CI-only users and did not use EAS as they considered it too cumbersome to wear 2 different devices (speech
Table 1. Inclusion criteria of M-Electrode and FLEX ${ }^{\mathrm{EAS}}$ study subjects

Postlingual, nonprogressive sensorineural hearing loss

Monosyllable scores in quiet of $\leq 45 \%$ (M-Electrode study) or $\leq 50 \%$ (FLEX $^{\mathrm{EAS}}$ study) at $65 \mathrm{~dB}$ SPL in best-aided condition (Oticon Adapto P behind-the-ear hearing aid)

Pure-tone hearing levels:

125, 250 and $500 \mathrm{~Hz}: \leq 65 \mathrm{~dB} \mathrm{HL}$

$1,000 \mathrm{~Hz}: \geq 60 \mathrm{~dB}$ HL (M-Electrode study) or $\geq 50 \mathrm{~dB} \mathrm{HL}$

(FLEX $^{\mathrm{EAS}}$ study)

2,000-8,000 Hz: $\geq 70 \mathrm{~dB} \mathrm{HL}$

processor and in-the-ear hearing aid) in the same ear. After the upgrade, all subjects did not only perform equally well or better in the objective speech perception tests, but showed also a tendency towards fewer problems with the DUET EAS system in the subjective APHAB questionnaire. Furthermore, all subjects remained EAS users with the DUET system. This suggests that the subjective benefit cannot only be attributed to the improved speech perception, but was also greatly influenced by the convenient fact that the EAS users were no longer required to wear 2 devices in the same ear but only one single device.

The present study focuses even more specifically on the subject benefits of EAS and assesses the subjective benefits reported by EAS users over time using the APHAB.

\section{Subjects and Methods}

Subjects

Twenty-three subjects with bilateral, symmetric hearing loss were enrolled in this investigation. Subjects were initially participants of 2 clinical trials which investigated the outcomes of EAS $[7,17]$. The inclusion criteria were the same in both studies (table 1), and each study site followed the same surgical protocol for atraumatic electrode insertion and the same recommended insertion depth of 18-22 mm. In both studies two different electrodes, however, both specifically designed for EAS treatment by MED-EL (Innsbruck, Austria) were used, namely the M-Electrode and the FLEX ${ }^{\mathrm{EAS}}$ electrode. Both electrodes feature 12 stimulation channels with contact spacing of $1.9 \mathrm{~mm}$ resulting in a total contact extent of $20.9 \mathrm{~mm}$.

Subjects from the FLEX ${ }^{\mathrm{EAS}}$ study were fit with the DUET EAS system which was the first device on the market that provided electric stimulation via the CI and acoustic amplification of audible low-frequency sounds in one combined speech processor. Subjects from the M-Electrode study were fit with the TEMPO+ 
speech processor and a separate in-the-ear hearing aid (Oticon Adapto-P, Oticon, Denmark) which was added to the same ear (as no DUET EAS system was available at the time of this study).

Eighteen subjects from the FLEX ${ }^{\mathrm{EAS}}$ study and, additionally, 5 subjects from the M-Electrode study completed the APHAB questionnaire and were included in this analysis.

\section{Methods}

Preoperative Assessment

Before implantation, all subjects used their preferred, everyday hearing condition. This means that some used hearing aids in both ears, some used a hearing aid in one ear and no acoustic amplification in the other ear, and some used no acoustic amplification at all in either ear. Thus, when answering the APHAB questions preoperatively, the subjects' hearing conditions were those used in everyday life.

\section{CI and Hearing Aid (EAS) Fitting}

The subjects were initially fitted with their speech processor (TEMPO+ or DUET) 3-5 weeks after cochlear implantation. The processor was fitted for the full frequency range available to provide adequate experience with electric stimulation via the $\mathrm{CI}$ alone. After 2 months, the acoustic amplification was added to the same ear, and thus the EAS mode was introduced to all subjects. The CI was fitted by determining at which frequency the audiogram surpassed $65 \mathrm{~dB}$ HL hearing loss. The low-frequency cutoff point of the electrical stimulation was set at this frequency point. The upper limit was set at 7,000 Hz. In both studies the acoustic stimulation was fitted in order to only amplify the audible low frequencies up to the frequency point where the audiogram reached $80 \mathrm{~dB}$ HL. For the M-Electrode group using a conventional hearing aid, fitting was performed using the Oticon Genie fitting software.

\section{Postoperative Assessments}

As described above, each subject completed 2 months of CIalone experience before the EAS mode was introduced. Thereafter, subjects had to complete the APHAB at EAS fitting as well as 3, 6 and 12 months after EAS fitting. In all postoperative test intervals when answering the APHAB questions, subjects referred to their hearing condition using EAS in the implanted ear and the hearing condition as used in everyday life in the contralateral ear, i.e. using a hearing aid or no acoustic amplification.

Detailed information about the objective speech perception tests performed in the two clinical trials combined in this study can be found in the papers published by Helbig et al. [17] and Gstoettner et al. [7].

\section{Abbreviated Profile of Hearing Aid Benefit}

In the APHAB $[12,13]$, subjects report about the amount of trouble they are having with communication and noise in everyday situations, i.e. the APHAB measures the percentage of difficulty experienced by subjects. The APHAB comprises 24 items that are divided into 4 subscales and summarizes these in a global scale. 'Ease of communication (EC)' is defined as the strain of communicating under relatively favorable conditions. 'Reverberation (RV)' considers communication in reverberant rooms such as classrooms, whilst 'background noise (BN)' considers communication in settings with high background noise levels. 'Aversiveness (AV)' evaluates the unpleasantness of environmental sounds.
Each item of the APHAB is a statement, and the subject must choose whether this statement is true by choosing from 7 options, ranging from 'A = always (99\%)' to ' $G=$ never (1\%)'. Several of the statements are presented in reverse order so that subjects focus on the content of the questions. A detailed description on the administration and application is available in the paper of Cox [13].

Statistical Analyses

Descriptive statistics were used to report baseline characteristics (e.g. age and gender). Quantitative data are presented as mean and range (minimum and maximum), and qualitative data as absolute and relative frequencies. The data distribution of the APHAB global scale and the 4 APHAB subscales (EC, RV, BN and $\mathrm{AV}$ ) are shown in graphs (box plots). A value of $100 \%$ reflects the highest possible impairment, i.e. the higher the percentage, the more subjectively reported problems. The subjective benefit of EAS was measured using the APHAB at different test intervals after implantation and compared to the preoperative acoustic hearing aid condition. One-way repeated-measures ANOVAs (GLM) with time as factor were performed on the APHAB global score and the APHAB subscales (EC, RV, BN and $\mathrm{AV}$ ) to investigate if significant improvement over time occurred. For each ANOVA, Mauchly's test of sphericity was applied. If sphericity could not be assumed, the Greenhouse-Geisser correction was used as part of the ANOVA. The Kolmogorov-Smirnov test was used to check the data distribution. To detect differences between the test intervals (difference from the preoperative testing to the first fitting, first fitting to the 3-month test interval, from the 3 -month to the 6-month test interval, and from the 6-month to the 12 -month test interval) parametric paired-sample $t$ tests were applied.

To determine the clinical relevance of EAS, a benefit score of the APHAB was assessed, according to the method of Cox and Alexander [12]. The benefit score was calculated by subtracting the aided average (e.g. first fitting) from the unaided average (e.g. preoperative testing). If the difference in benefit scores on the 3 subscales EC, RV and $\mathrm{BN}$ were at least $10 \%$ (difference in mean) greater for the respective test strategy, it can be concluded from the clinical perspective that this difference reflects a true benefit with a $95 \%$ probability.

Statistical significance was set to $p<0.05$. When considering Bonferroni's adjustment for multiple comparisons, $p$ values less than 0.0125 indicate statistical significance for 4 pairwise tests. SPSS 16.0 for Windows software (Chicago, Ill., USA) was used for all analyses. Graphs were created in Microsoft Office Excel 2003.

Ethics

This study was conducted according to EN 540 standards (the standards in operation at the time of the M-Electrode study) and ISO 14155 parts 1 and 2 for the FLEX ${ }^{\text {EAS }}$ study. Ethics approval for the study was received from each participating institution, and all relevant competent authorities approved the study.

\section{Results}

Data of 23 EAS users implanted with either the FLEX ${ }^{\text {EAS }}$ or the M-Electrode were analyzed in this study. Twelve women (67\%) and 6 men (28\%) were included from the 
Fig. 1. $A P H A B$ global score: subjectively reported problems (black squares: mean; horizontal stripes: median) for patients using EAS over time. A value of $100 \%$ reflects the highest possible impairment.
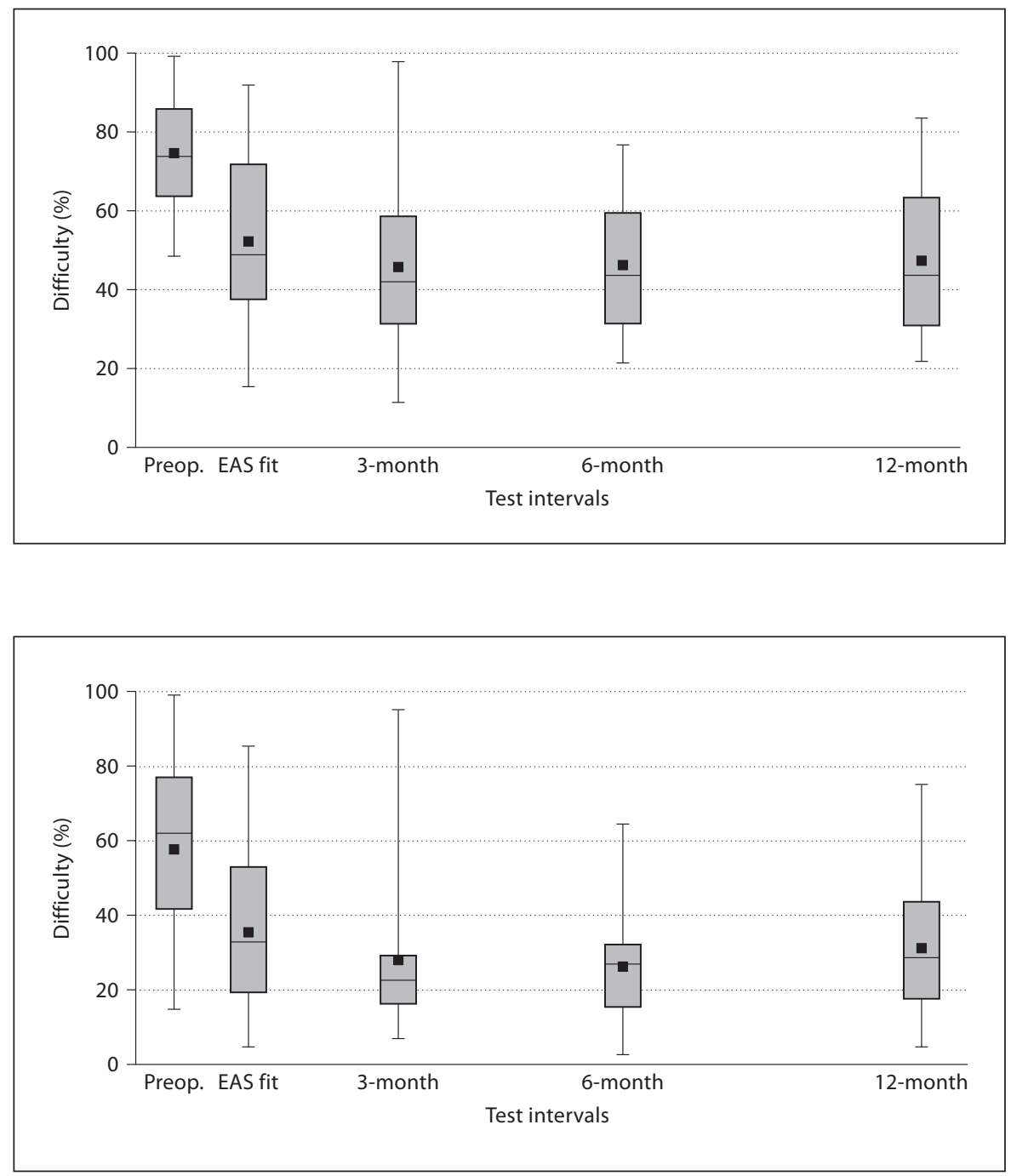

Fig. 2. $A P H A B$ EC subscale: subjectively reported problems (black squares: mean; horizontal stripes: median) for patients using EAS over time. A value of $100 \%$ reflects the highest possible impairment.
FLEX $^{\text {EAS }}$ electrode study [17]. The mean age at surgery was 51 years, with a range of $22-75$ years. Nine patients (50\%) were implanted in the left ear and 9 patients (50\%) in the right. The mean age at onset of profound hearing loss for both ears was 44 years, with a range of 12-66 years. The mean duration of deafness at assessment time was 20 years (range: $5-38$ years). One woman (20\%) and 4 men $(80 \%)$ were included from the M-Electrode study [7]. The mean age at the time of surgery was 57 years, ranging from 48 to 69 years. Four subjects were implanted in the left ear and 1 in the right. The mean age at onset of profound hearing loss was 43 years (range: 25-61 years), and the mean duration of deafness was 24 years (range: $11-54$ years).

In figures 1-5, mean $\mathrm{APHAB}$ percentage scores for the global score and for the $\mathrm{EC}, \mathrm{RV}, \mathrm{BN}$ and $\mathrm{AV}$ subscales are shown for the different test intervals. The measured mean percentage of problems decreased from $74 \%$ before implantation to $45 \%$ after 3 months of EAS use for the global scale, from 57 to $28 \%$ for the EC subscale, from 83 to $55 \%$ for the RV subscale, from 82 to $53 \%$ for the BN subscale and from 45 to $27 \%$ for the AV subscale.

According to the results of one-way repeated-measures ANOVAs, the improvement over time with the EAS condition compared to the preoperative acoustic hearing aid condition for the global score and for all subscales was statistically highly significant $[\mathrm{p}<0.001$; global score: $\mathrm{F}(2.5,46.9)=26.91$; EC: $\mathrm{F}(2.3,42.9)=15.20$; RV: $\mathrm{F}(2.7$, $50.6)=16.48 ; \mathrm{BN}: \mathrm{F}(2.6,49.4)=22.01 ; \mathrm{AV}: \mathrm{F}(1.9,35.8)=$ 3.35; table 2].

Statistical analyses investigating the differences between the single test intervals revealed significant im- 
Fig. 3. $A P H A B$ RV subscale: subjectively reported problems (black squares: mean; horizontal stripes: median) for patients using EAS over time. A value of $100 \%$ reflects the highest possible impairment.

Fig. 4. $A P H A B B N$ subscale: subjectively reported problems (black squares: mean; horizontal stripes: median) for patients using EAS over time. A value of $100 \%$ reflects the highest possible impairment.

Fig. 5. APHAB AV subscale: subjectively reported problems (black squares: mean; horizontal stripes: median) for patients using EAS over time. A value of $100 \%$ reflects the highest possible impairment.
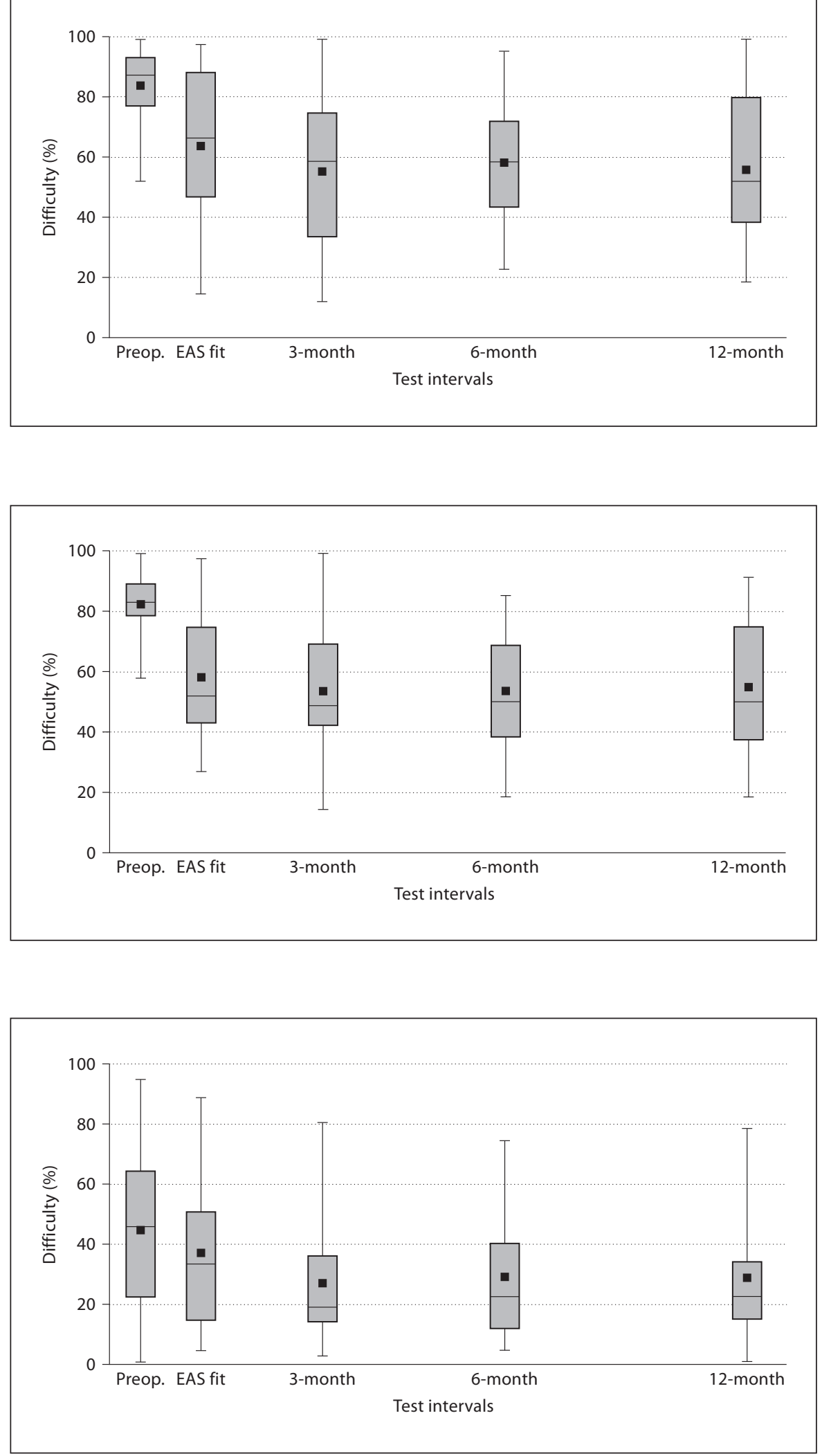
Fig. 6. Preoperative results (= hearing aid condition), first fitting and 12-month testing results of Hochmair-Schulz-Moser sentences in noise depicted separately for the whole sample (all: $\mathrm{n}=23$ ), the FLEX ${ }^{\mathrm{EAS}}$ sample $(\mathrm{n}=18)$ and the M-Electrode sample $(n=5)$. Speech was presented at a level of $65 \mathrm{~dB}\left(\right.$ FLEX $^{\mathrm{EAS}}$ [17]) and $70 \mathrm{~dB}$ (MElectrode [7]). The signal-to-noise ratio was set at $10 \mathrm{~dB}$. Horizontal lines of the box plot represent median values, black squares mean values.

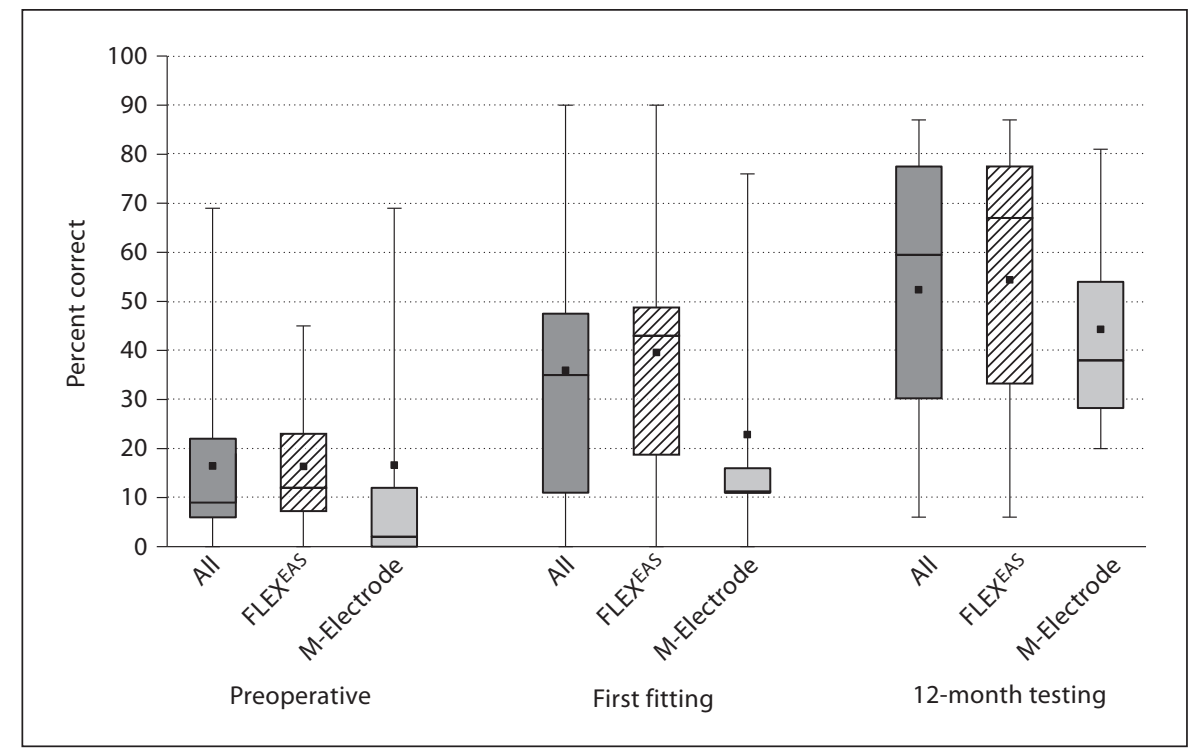

Table 2. Results of paired-sample t tests to detect mean differences between the single test intervals for the APHAB global score and the EC, RV, BN and AV subscales

\begin{tabular}{lclll}
\hline & $\begin{array}{l}\text { Preop. vs. } \\
\text { EAS fit }\end{array}$ & $\begin{array}{l}\text { EAS fit vs. } \\
3 \text { months }\end{array}$ & $\begin{array}{l}\text { 3 vs. } 6 \\
\text { months }\end{array}$ & $\begin{array}{c}6 \text { vs. 12 } \\
\text { months }\end{array}$ \\
& & & & \\
Global & & & & \\
$\quad \begin{array}{l}\text { Difference } \\
\text { p value (2-tailed) }\end{array}$ & $<0.001$ & 0.001 & 0.768 & 0.405 \\
EC & & & & \\
$\quad$ Difference & 23.7 & 8.6 & 1.0 & -5.2 \\
p value (2-tailed) & 0.001 & 0.014 & 0.700 & 0.018 \\
RV & & & & \\
Difference & 21.7 & 9.1 & -2.1 & 2.1 \\
p value (2-tailed) & $<0.001$ & 0.001 & 0.409 & 0.487 \\
BN & & & & \\
$\quad$ Difference & 24.7 & 6.0 & -0.6 & -1.3 \\
p value (2-tailed) & $<0.001$ & 0.042 & 0.790 & 0.513 \\
AV & & & & \\
$\quad$ Difference & 7.2 & 9.4 & -2.5 & 0.6 \\
p value (2-tailed) & 0.192 & 0.015 & 0.413 & 0.826 \\
\hline
\end{tabular}

Significant results of $\mathrm{p}<0.0125$ are in italics.

provement for the global score and almost all subscales between the preoperative testing and the first fitting, and between the first fitting and the 3-month testing. The improvement for the AV subscale between the preoperative testing and the first fitting, and for the BN subscale between first fitting and 3-month testing was not signifi- cant. Also, between the 3-month and 6-month testing and between the 6-month and 12-month testing, the difference was not statistically significant for the global score and the subscales, except for the EC subscale (table 2).

Using the method of Cox and Alexander [12] to evaluate the clinical relevance of the device, the mean differences in benefit scores on the 3 subscales EC, RV and BN between preoperative and first fitting results were higher than $10 \%(22,20$ and $24 \%$, respectively) and thus reflected with $95 \%$ probability a true benefit of EAS. The AV subscale and comparisons between the preoperative and the 6-month as well as the 12-month interval showed a tendency to improved benefit but the results were not significant from a clinical perspective according to the method of Cox and Alexander [12].

\section{Discussion}

The present study used the APHAB questionnaire to assess the subjective benefits of EAS over the first 12 months after EAS fitting. Although this field of application was initially not intended for the APHAB as it was validated only for use in individuals using hearing aids [12], multiple studies in the past have already demonstrated the applicability of the APHAB questionnaire for investigating the subjective benefits of CIs $[14,18]$ and EAS $[16,17,19]$. Against this backdrop, we considered the APHAB to be a valid testing method for evaluating the subjective benefits of EAS. 


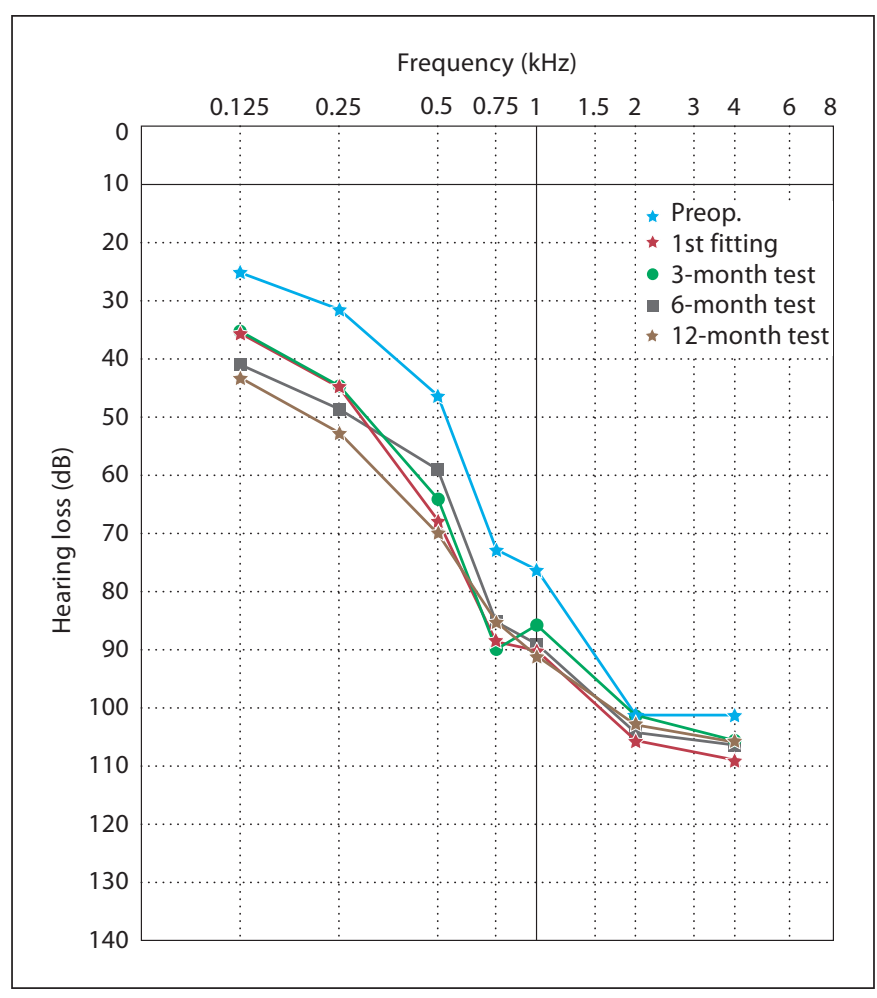

Fig. 7. Mean hearing threshold levels of all subjects.

The results of the APHAB show that subjects perceived an immediate benefit with EAS with statistically significant differences between the acoustic-only amplification pre-operatively and the EAS at the first fitting as well as between the first fitting and the 3-month interval in the global scale and almost all subscales. After this statistically significant decrease in impairment, the scores stabilized following the 3-month interval at this low level of impairment (fig. 1-5). These results correspond with the objective speech perception results (fig. 6) [for more detailed speech perception results and test conditions, see also 7, 17]. Figure 6 shows the improvement in speech perception over time in the Hochmair-Schulz-Moser sentence test in noise. We decided to use the HochmairSchulz-Moser sentence test for a direct comparison here as it is the test closest to a 'real-life' situation and is thus best to be compared with the APHAB, in which the impaired patients report their amount of trouble with communication and noise in everyday situations. The improvement was statistically significant for the combined groups [repeated-measure ANOVAs: $\mathrm{F}(2,38)=11.81$; $\mathrm{p}<$ $0.001]$ and for the FLEX ${ }^{\mathrm{EAS}}$ group $[\mathrm{F}(2,30)=12.07$; $\mathrm{p}<$ $0.001]$ but not for the M-Electrode group $[\mathrm{F}(2,6)=2.85$; $p=0.237]$. Furthermore, the difference was statistically significant between the preoperative and the first fitting interval $(p=0.008)$ and between the first fitting and the 12 -month interval $(p=0.021)$. Thus, the APHAB results as well as the speech perception results correlate very well and demonstrate an immediate benefit of EAS, suggesting that the APHAB is a suitable method of measuring subjective benefits of EAS. However, in contrast to the objective speech perception results, which improved over a longer period of time (Hamzavi et al. [20]: up to 72 months; Tyler et al. [21]: up to 18-30 months), suggesting that more time to get accustomed to a device and/or a new coding strategy further increases speech perception, the APHAB results in our study stabilized after the 3-month interval, which might be attributed to fulfilled expectations.

It is furthermore interesting to note that this immediate benefit, especially the benefit between the preoperative and first fitting interval, could be observed considering that all subjects experienced a drop in the threshold levels after surgery at the critical frequencies of 125, 250 and $500 \mathrm{~Hz}$ (fig. 7). Subjects had a mean hearing loss of $12 \mathrm{~dB}(\mathrm{SD}=11)$ at $125 \mathrm{~Hz}$, of $14 \mathrm{~dB}(\mathrm{SD}=12)$ at $250 \mathrm{~Hz}$ and of $22 \mathrm{~dB}(\mathrm{SD}=17)$ at $500 \mathrm{~Hz}$ between preoperative testing and first fitting. After this initial drop, the residual hearing was stable over time and therefore should not have had a further influence on the results presented here (fig. 7). Our results suggest that EAS users have relevant subjective and objective benefit from the treatment even if some hearing is lost postoperatively.

Similar APHAB results, showing an immediate benefit, are also reported in an EAS study by Skarzynski et al. [5]. In the APHAB global scores, subjects showed statistically significant differences between the preoperative and the 6 -month intervals $(\mathrm{p}=0.085)$ as well as between the preoperative and the 12 -month intervals $(\mathrm{p}=0.087)$, but not between the 6- and the 12-month intervals. According to Skarzynski et al., the level of (direct) benefit reported by subjects may also reflect if the fitting parameters correspond with the individual's needs or if changes in fitting have to be made. In their study, the results in the AV subscales worsened within the first months after implantation. Skarzynski et al. related this increased impairment to the fact that the subjects were not accustomed to the sound and that the fitting parameters put too much emphasis on the high frequencies. In our study, an improvement in the AV condition was still observable, although it was not statistically significant for the majority of test intervals. Nevertheless, the explanation by Skarzynski et al. [5] for the worsened results in their AV 
subscale, suggesting that time of adjustment and fitting parameters might have an influence on subjectively perceived benefits with EAS, might also be the reason for the only small, statistically not significant improvement in the given AV subscale results in our study. The APHAB thus allows not only to observe the degree of subjective benefit, but it can also be considered as a tool to assess the adjustment of different fitting parameters.

As mentioned previously, the APHAB was designed and used in this study for hearing-impaired patients to report the amount of trouble they are having with communication and noise in everyday life between the preoperative and postoperative situations. Generally, it would also be interesting to compare EAS APHAB results not only with preoperative results, but also with subjective results in the CI condition. Many studies have shown the benefits of EAS in comparison to electric stimulation alone with various types of speech and music tests (see Talbot and Hartley [22] for detailed study references). However, the analysis of superiority of EAS over electric stimulation only was not the aim of this study. Our focus was directed at demonstrating the subjectively reported benefits gained with EAS over time. We furthermore believe that it is not possible to compare the CI-only and EAS conditions within the same patient using the APHAB. For comparing APHAB results in CI and EAS conditions within the same patients, they would have to use both conditions for the same amount of time. However, this would be unethical as patients would be deprived from the use of a potentially better mode of stimulation. Additionally, this would not be possible in some patients without having to plug one or both ears over the whole testing period if their residual hearing was good enough to perceive acoustic information even without a hearing aid. Furthermore, it could produce bias concerning the experience with electric stimulation.
The only valuable possibility to assess superiority of EAS over electric stimulation only using the APHAB would be to compare two groups of patients according to their postoperative residual hearing status: (1) patients with preserved residual hearing, i.e. EAS users, and (2) patients who lost all their residual hearing during surgery, i.e. CI-only users.

In our study, only those subjects from both clinical trials $[7,17]$ who had preserved residual hearing, and thus EAS users, were included. Subjects with loss of residual hearing, and thus CI-only users, were not followed up with the APHAB. This might be interpreted as a weakness of this study as no comparison of APHAB results between CI and EAS users was done. However, as stated above, it was not the objective of this study to investigate the superiority of EAS over CI-only. At the same time, this aspect is undoubtedly a topic of major interest and should be investigated in future studies.

\section{Conclusion}

The excellent improvements in the global scale and the subscales as well as the clinical significance suggest that EAS provides great subjective benefit immediately as well as over time and compared to acoustic amplification only.

\section{Acknowledgements}

The authors gratefully acknowledge Edda Amann for statistical analyses, Denise Shepherd for data management as well as Jasmin Stadel for her writing assistance, all on behalf of MED-EL.

\section{Disclosure Statement}

The authors report no conflicts of interest related to this study.

\section{References}

1 Von Ilberg C, Kiefer J, Tillein J, Pfenningdorff T, Hartmann R, Sturzebecher E, Klinke R: Electric-acoustic stimulation of the auditory system. New technology for severe hearing loss. ORL J Otorhinolaryngol Relat Spec 1999;61:334-340.

-2 Kiefer J, Gstoettner W, Baumgartner W, Pok S, Tillein J, Ye Q, Von Ilberg C: Conservation of low frequency hearing in cochlear implantation. Acta Otolaryngol 2004;124:272280.

\footnotetext{
-3 Skarzynski H, Lorens A, Piotrowska A, Anderson I: Preservation of low frequency hearing in partial deafness cochlear implantation (PDCI) using the round window surgical approach. Acta Otolaryngol 2007;127: 41-48.

-4 Gstoettner W, Kiefer J, Baumgartner W, Pok S, Peters S, Adunka O: Hearing preservation in cochlear implantation for electric acoustic stimulation. Acta Otolaryngol 2004; 124: 348-352.
}

\footnotetext{
5 Skarzynski H, Lorens A, Piotrowska A, Anderson I: Partial deafness cochlear implantation provides benefit to a new population of individuals with hearing loss. Acta Otolaryngol 2006;126:934-940.

-6 Fraysse B, Burdo S, Ramsden R, Deguine O, Klenzner T, Lenarz T, Rodriguez M, Von Wallenberg E, James C: Residual hearing conservation and electroacoustic stimulation with the Nucleus 24 contour advance cochlear implant. Otol Neurotol 2006;27: 633.
} 
7 GstoettnerWK, Van de Heyning P, O'Connor AF, Morera C, Sainz M, Vermeire K, McDonald S, Cavalle L, Helbig S, Valdecasas JG, Anderson I, Adunka OF: Electric acoustic stimulation of the auditory system: results of a multi-centre investigation. Acta Otolaryngol 2008;12:1-8.

-8 Brockmeier SJ, Peterreins M, Lorens A, Vermeire K, Helbig S, Anderson I, Skarzynski H, Van de Heyning P, Gstoettner W, Kiefer J: Music perception in electric acoustic stimulation users as assessed by the MuSIC test. Adv Otorhinolaryngol 2010;67:70-80.

$\checkmark 9$ Cox RM, Gilmore C, Alexander GC: Comparison of two questionnaires for patient-assessed hearing aid benefit. J Am Acad Audiol 1991;134-145.

10 Driver S, Stark E: Real life outcomes for adult EAS patients and adult traditional CI patients. Cochlear Implants Int 2010;11(suppl 2):113-118.

11 Gatehouse S: Glasgow hearing aid benefit profile: derivation and validation of a clientcentered outcome measure for hearing aid services. J Am Acad Audiol 1999;10:80-103.
12 Cox RM, Alexander GC: The abbreviated profile of hearing aid benefit. Ear Hear 1995; 16:176-186.

13 Cox R: Administration and Application of the APHAB. Hear J 1997;50:32-48.

14 Litovsky R, Parkinson A, Arcaroli J, Sammeth C: Simultaneous bilateral cochlear implantation in adults: a multicenter clinical study. Ear Hear 2006;27:714-731.

15 Valente M, Mispagel KM: Unaided and aided performance with a directional open-fit hearing aid. Int J Audiol 2008;47:329-336.

16 Helbig S, Baumann U, Helbig M, von MalsenWaldkirch N, Gstoettner W: A new combined speech processor for electric and acoustic stimulation - eight months experience. ORL J Otorhinolaryngol Relat Spec 2008;70:359-365.

17 Helbig S, Van de Heyning P, Kiefer J, Baumann U, Kleine-Punte A, Brockmeier $\mathrm{H}$ Anderson I, Gstoettner W: Combined electric acoustic stimulation with the PULSAR $\mathrm{CI}^{100}$ implant system using the FLEX ${ }^{\mathrm{EAS}}$ electrode array. Acta Otolaryngol 2011;131: 585-595.
8 Plyler PN, Bahng J, von Hapsburg D: The acceptance of background noise in adult cochlear implant users. J Speech Lang Hear Res 2008;51:502-515.

19 Gifford R, Dorman M, Spahr A, McKarns S: Effect of digital frequency compression (DFC) on speech recognition in candidates for combined electric and acoustic stimulation (EAS). J Speech Lang Hear Res 2007;50: 1194-1202.

$>20$ Hamzavi J, Baumgartner W, Pok S, Franz P, Gstöttner W: Variables affecting speech perception in postlingually deaf adults following cochlear implantation. Acta Otolaryngol 2003;123:493-498.

-21 Tyler R, Parkinson A, Woodworth G, Lowder M, Gantz B: Performance over time of adult patients using the Ineraid or Nucleus cochlear implant. J Acoust Soc Am 1997;102:508522.

22 Talbot KN, Hartley DE: Combined electroacoustic stimulation: a beneficial union? Clin Otolaryngol 2008;33:536-545. 